\title{
Reduced-Order Nonlinear Unsteady Aerodynamic Modeling Using a Surrogate-Based Recurrence Framework
}

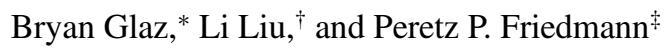 \\ University of Michigan, Ann Arbor, Michigan 48109
}

DOI: $10.2514 / 1 . J 050471$

\begin{abstract}
A reduced-order nonlinear unsteady aerodynamic modeling approach suitable for analyzing pitching/plunging airfoils subject to fixed or time-varying freestream Mach numbers is described. The reduced-order model uses kriging surrogates to account for flow nonlinearities and recurrence solutions to account for time-history effects associated with unsteadiness. The resulting surrogate-based recurrence framework generates time-domain predictions of unsteady lift, moment, and drag that accurately approximate computational fluid dynamics solutions, but at a fraction of the computational cost. Results corresponding to transonic conditions demonstrate that the surrogate-based recurrence framework can mimic computational fluid dynamics predictions of unsteady aerodynamic responses when flow nonlinearities are present. For an unsteady aerodynamic modeling problem considered in this study, an accurate reduced-order model was generated by the surrogate-based recurrence framework approach with significantly fewer computational fluid dynamics evaluations compared to results reported in the literature for a similar problem in which a proper-orthogonal-decomposition-based approach was applied. Furthermore, the results show that the surrogate-based approach can accurately model time-varying freestream Mach number effects and is therefore applicable to rotary-wing applications in addition to fixed-wing applications.
\end{abstract}

\section{Nomenclature}

$a$
$b$
$C_{l}, C_{m}, C_{d}$
$E^{(j)}$
$\mathbf{F}$

$f_{x}$

$G_{q}, G_{y}$

$\underline{h}$

$\bar{h}$

$k_{h}$

$k_{M}$

$k_{\theta}$

$L$

$M$

$\bar{M}$

$M_{0}$

$m, n$

$N_{\text {basis }}$

$N_{\text {sp }}$

$N_{T}$

$N_{t}$

$N_{x}$

$p_{k}$

$=$ speed of sound

$=$ airfoil semichord

$=$

$=$ error for the $j$ th test case

$=$ polynomials in kriging polynomials in kriging mapping function

$=$ reduced plunge frequency

$=$ reduced pitch frequency

$=$ freestream Mach number

$=$ number of sample points

$=$ number of training cases

$=$ number of input variables airfoil lift, moment, and drag coefficients

$=$ vector of basis functions associated with assumed

$=$ state transition function and state-to-output

$=$ airfoil plunge degree of freedom

$=$ oscillatory plunge amplitude

$=$ reduced frequency of time-varying Mach number

$=$ likelihood function used in kriging interpolation

$=$ time-varying Mach number amplitude

$=$ mean value of time-varying Mach number

$=$ number of previous time steps required to account for time-history effects [see Eq. (4)]

$=$ number of basis functions associated with assumed polynomials in kriging

$=$ total number of time steps for a test case

$=$ fitting parameters in kriging q

$R$

$\mathbf{R}_{\mathrm{krg}}$

$R_{\mathrm{krg}}(\cdot)$

$r$

$\mathbf{r}_{\mathrm{krg}}(\mathbf{x})$

$\mathbf{u}$

$V_{0}$

$\hat{y}$

$\hat{y}_{y_{\max }}^{(j)}, y_{\min }^{(j)}$

$Z(\mathbf{x})$

$\underline{\theta}$

$\bar{\theta}$

$\mu$

$\hat{\Phi}$

$\hat{\Phi}_{\mathrm{qs}}$

\section{$\Omega$}

$\omega_{h}$

$\omega_{M}$

$\omega_{\theta}$
$=$ dynamical system state vector

$=$ rotor radius

$=$ spatial correlation matrix used in kriging

$=$ spatial correlation function in kriging

$=$ nondimensional rotor blade radial location

$=$ spatial correlation vector in kriging

$=$ vector of external inputs

$=$ mean value of freestream velocity

$=$ output of interest

$=$ surrogate approximation of output of interest

$=$ maximum and minimum values of the exact response for the $j$ th test case

$=$ stochastic process in kriging

$=$ airfoil pitch angle

$=$ oscillatory pitch amplitude

$=$ advance ratio

$=$ nonlinear mapping function

$=$ surrogate mapping function

$=$ quasi-steady mapping function used for surrogate-based recurrence framework initialization

$=$ rotational speed of the rotor

$=$ oscillatory plunge frequency

$=$ oscillatory frequency of time-varying Mach number

$=$ oscillatory pitch frequency

Received 1 February 2010; revision received 24 June 2010; accepted for publication 2 July 2010 . Copyright $\odot 2010$ by Bryan Glaz, Li Liu, and Peretz P. Friedmann. Published by the American Institute of Aeronautics and Astronautics, Inc., with permission. Copies of this paper may be made for personal or internal use, on condition that the copier pay the $\$ 10.00$ per-copy fee to the Copyright Clearance Center, Inc., 222 Rosewood Drive, Danvers, MA 01923; include the code 0001-1452/10 and $\$ 10.00$ in correspondence with the CCC.

*AGEP Postdoctoral Research Fellow, Department of Aerospace Engineering. Senior Member AIAA.

Postdoctoral Research Fellow, Department of Aerospace Engineering. Senior Member AIAA

François-Xavier Bagnoud Professor, Department of Aerospace Engineering. Fellow AIAA.

\section{Introduction}

$\mathbf{U}$ NSTEADY aerodynamic models are critical components in computational aeroelastic response and stability analyses. In addition to the time-history effects associated with unsteadiness, many computational aeroelasticity applications also involve strongly nonlinear aerodynamic phenomena, such as effects associated with transonic flow, hypersonic flow, and dynamic stall. Furthermore, unsteady aerodynamic modeling is complicated further for rotarywing applications due to the effects of time-varying freestream Mach numbers in forward flight [1] .

Currently, computational fluid dynamics (CFD) solutions represent the state of the art in modeling nonlinear flow physics. However, due to the excessive computational cost, high-fidelity CFD simulations are not suitable for a variety of applications, such as routine 
coupled trim/aeroelastic response solutions, design optimization, and/or closed-loop control studies. Therefore, computationally efficient approximate aerodynamic models are typically employed in such studies. Representative examples of the approximate models employed in rotary-wing applications are the rational function approximation (RFA) approach [2] and the indicial function approach [3]. Although such models require a fraction of the computational expense associated with CFD, they are based on various simplifying assumptions pertaining to the flow physics. For example, the RFA and indicial function models are based on linear aerodynamic assumptions, and therefore both models cannot accurately capture nonlinear flow phenomena associated with transonic flow and/or dynamic stall.

In addition to developing computationally efficient aerodynamic models based on simplifying assumptions, reduced-order modeling approaches that seek to approximate CFD results by extracting information from a limited number of full-order simulations have been proposed. Examples of such approaches include proper orthogonal decomposition (POD) [4-7], Volterra series [4,8], and surrogate-based approaches [9-13]. A major advantage of reducedorder modeling of full-order $\overline{\mathrm{C} F \mathrm{D}}$ compared to approaches based on simplifying assumptions is that the reduced-order models can be easily upgraded as the CFD solvers from which they are generated continue to improve. However, the reduced-order models described in previous studies have certain shortcomings when attempting to model the rotary-wing aerodynamic environment. Specifically, the effectiveness of current reduced-order modeling approaches for producing an accurate-time-domain, fully unsteady model that accounts for simultaneous pitch/plunge airfoil motions and timevarying freestream Mach numbers has not been considered. Furthermore, rotor blade dynamics involve large unsteady oscillations of the airfoil motion and freestream Mach number, as opposed to many fixed-wing aeroelasticity applications in which reduced-order aerodynamic models only need to account for small amplitude dynamics associated with perturbations about a linearized steady state. The lack of nonlinear reduced-order aerodynamic modeling approaches suitable for rotary-wing aeroelasticity has been noted in previous studies [14,15].

The overall objective of this study is to develop a CFD-based reduced-order nonlinear aerodynamic model suitable for rotary-wing analyses. It is important to note that while the complex rotary-wing aerodynamic environment motivated the development of the model described in this paper, the approach is general and can be used in a variety of applications involving unsteady aerodynamics. The specific objectives of this paper are listed below.

1) Develop a two-dimensional (2-D) reduced-order aerodynamic modeling approach based on surrogate modeling [16-18] that accurately approximates CFD predictions corresponding to arbitrary airfoil motions.

2) Demonstrate the effectiveness of the surrogate-based framework by showing that such a methodology enables unsteady sectional load (lift, moment, and drag) predictions, for fixed or timevarying Mach numbers, that maintain the fidelity of full-order CFD solutions under nonlinear transonic flow conditions, while exhibiting the computational efficiency of approximate linear models.

\section{Surrogate-Based Reduced-Order Model}

Surrogates refer to computationally efficient approximations of expensive functions that are constructed by interpolating fitting data in the form of input/output combinations generated from a limited number of full-order computations [16-18]. Once constructed, a surrogate is used in place of the expensive full-order analysis in order to predict at inputs that were not included in the initial set of fitting points. Typical surrogate prediction times are on the order of a fraction of a second, and therefore surrogates are ideal for reducedorder modeling. Although generating the fitting data may require significant computational resources, this initial cost is generally much less than computing repeated solutions from the expensive fullorder analysis. Therefore, surrogate-based approaches are appropriate for applications that entail numerous full-order analysis evaluations that would otherwise be unaffordable. A variety of computational aeroelasticity and design optimization applications involving CFD fall under this category.

In this study, CFD solutions for unsteady lift, moment, and drag due to an arbitrary airfoil motion represent the full-order analysis that is to be replaced by surrogates. The methodologies for generating and using the surrogates for time-domain predictions are described next.

\section{A. Dynamical System Representation of the Input/Output Relationship}

The input/output relationship that is to be approximated by a surrogate model can be identified by formulating unsteady aerodynamic responses as dynamical systems: i.e., systems in which the output at any time $t$ is not only a function of the instantaneous input at $t$, but is also a function of the input time history. A general representation of a nonlinear time-invariant discrete time dynamical system is given by [19-22]

$$
\mathbf{q}(t)=G_{q}(\mathbf{q}(t-\Delta t), \mathbf{u}(t-\Delta t)), \quad y(t)=G_{y}(\mathbf{q}(t))
$$

where $\mathbf{q}$ is a vector containing the system states, $\mathbf{u}$ is the vector of external inputs to the system, $G_{q}$ is the one-step-ahead state transition function, $y(t)$ is the output of interest at some instant in time $t$, and $G_{y}$ is a function that maps the system states to the output.

In the context of unsteady aerodynamic modeling, the nonlinear system of equations described by Eq. (1) represents the discretized Navier-Stokes equations associated with CFD solvers. The state vector $\mathbf{q}$ consists of the flow states associated with the Navier-Stokes equations (i.e., density, velocity components, and energy) at each grid point in the spatially discretized computational domain. Therefore, the size of the state vector is proportional to the number of grid points and represents the order of the full-order model. Furthermore,

$$
y(t) \equiv C_{l}(t), C_{m}(t), \quad \text { or } \quad C_{d}(t)
$$

where $C_{l}(t), C_{m}(t)$, and $C_{d}(t)$ are the unsteady airfoil lift, moment, and drag coefficients, respectively. The relevant external inputs are

$$
\mathbf{u}(t) \equiv\left[\begin{array}{lllllll}
\theta(t) & \dot{\theta}(t) & \ddot{\theta}(t) & \dot{h}(t) & \ddot{h}(t) & M(t) & \dot{M}(t)
\end{array}\right]
$$

As shown in Fig. 1, $\theta(t), h(t)$, and $M(t)$ are the instantaneous pitch angle, plunge displacement, and freestream Mach number, respectively. Time derivatives are denoted by $\dot{O} \equiv \partial() / \partial t$ and $\ddot{O} \equiv$ $\partial^{2}() / \partial t^{2}$. The inputs in Eq. (3) were selected because it is known from linear aerodynamic theories that unsteady lift and moment are functions of these quantities [23], including $\dot{M}$ when modeling timevarying Mach numbers []ㅡ (which is a requirement for rotary-wing applications).

Equation (1) represents the computationally expensive full-order system that we seek to replace with a surrogate-based reduced-order model. To generate a computationally efficient approximation of $y(t)$ using surrogate modeling, the dynamical system of interest must be replaced by an equivalent input/output functional relationship such that $y(t)$ corresponding to any input time history can be obtained
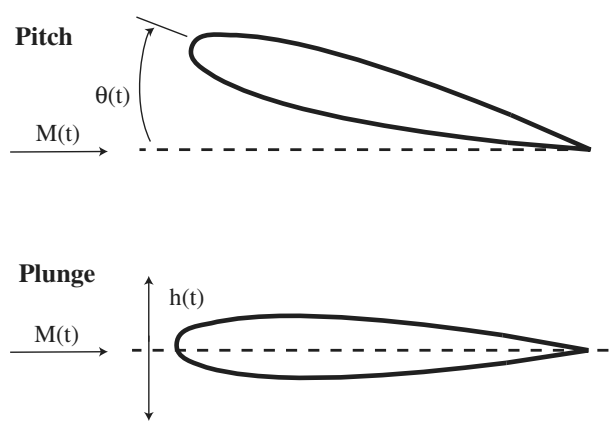

Fig. 1 Airfoil pitch and plunge degrees of freedom with time-varying freestream Mach number. 
without solving the nonlinear system of equations described by Eq. (1). It has been shown in [19-21] that when $\mathbf{q}$ is composed of a finite number of states, the input/output relationship given by Eq. (4) is equivalent to the nonlinear system described by Eq. (1):

$$
\begin{aligned}
& y(t)=\Phi(\mathbf{u}(t), \mathbf{u}(t-\Delta t), \ldots, \mathbf{u}(t-m \Delta t), y(t-\Delta t), \ldots, \\
& y(t-n \Delta t))
\end{aligned}
$$

In Eq. (4), $\Phi$ is a function-possibly nonlinear-that maps the inputs to the output, and $m$ and $n$ are integers representing the number of previous external inputs and outputs required to account for timehistory effects. Selection of $m$ and $n$ is discussed in Sec. IV. Equation (4) is commonly referred to as a NARMAX (nonlinear autoregressive moving average with exogeneous inputs) model $[19, \underline{21}, 22,24,25]$.

It is important to note that the derivations described in $[19,20]$ guarantee the validity of Eq. (4) only in the vicinity of an equilibrium state. An equilibrium state $\mathbf{q}_{0}$ is a state in which the system is at rest for an input sequence that has a constant value $\mathbf{u}_{0}$; i.e., $y(t)$ is constant for all $t$ when $\mathbf{q}=\mathbf{q}_{0}$ and $\mathbf{u}(t)=\mathbf{u}_{0}$. Therefore, according to [19,20], Eq. (4) is only guaranteed to be valid in the vicinity of $\mathbf{u}_{0}$. However, it was shown in [21] that Eq. (4) can also be used to describe a globally valid input/output relationship, and thus Eq. (4) is not restricted to modeling systems for $\mathbf{u}(t)$ close to $\mathbf{u}_{0}$. The globally valid model described in [21] is characterized by increased values of $m$ and $n$ required to capture time-history effects compared to the locally valid model described in $[\underline{19}, \underline{20}]$.

\section{B. Approximation of the Nonlinear Mapping Function}

Although Eq. (4) provides a qualitative representation of the input/ output relationship associated with a nonlinear dynamical system, it is of little practical use for modeling unsteady aerodynamic responses, because a closed-form expression for $\Phi$ is not available. However, $\Phi$ can be numerically evaluated, or sampled, by employing CFD as a black-box function that calculates lift, moment, and drag for a given airfoil motion time history. A surrogate mapping function $\hat{\Phi}$ can then be constructed by interpolating the sample data. Once constructed, the computationally efficient surrogate can be used to replace the full-order function $\Phi$. When modeling dynamical systems, the process of approximating the unknown function $\Phi$ is also referred to as system identification [22]. The steps for generating the surrogate mapping function are summarized below, and additional details for each step are provided in the following subsections.

1) Use design of experiment (DOE) methods $[17,26]$ to select a limited number of training cases at which to conduct CFD simulations. Each training case consists of an airfoil undergoing simultaneous pitch/plunge oscillations, with either a fixed freestream Mach number, or time-varying Mach numbers if the surrogate is to be used for rotary-wing applications.

2) Generate aerodynamic response data for each training case using CFD, and then extract time-domain sample data of the form dictated by Eq. (4) from the results. Each training case can be computed simultaneously using multiple processors. Therefore, the time required to generate the initial fitting data can be decreased significantly through the use of parallel computation.

3) Construct a surrogate mapping function $\hat{\Phi}$ from the sampled data by employing kriging interpolation $[27,28]$, which is a method well suited for approximating nonlinear functions.

\section{Selection of the Training Cases (DOE)}

To generate the input/output combinations required for interpolation, the unsteady aerodynamic responses corresponding to a limited number of training cases are obtained from CFD simulations. The training cases should be representative of the application for which the surrogate will be used. For instance, the training motions considered in this study correspond to simultaneous pitch/plunge oscillations with fixed or time-varying freestream Mach numbers, since these cases are representative of those encountered in rotary- wing aeroelasticity. The airfoil oscillations and time-varying Mach numbers used for training are defined by Eqs. (ㅁ-ㄱ):

$$
\begin{gathered}
\theta(t)=\bar{\theta} \cos \left(\omega_{\theta} t\right) \\
h(t)=\bar{h} \cos \left(\omega_{h} t\right) \\
M(t)=M_{0}+\bar{M} \sin \left(\omega_{M} t\right)
\end{gathered}
$$

Equation (7) is representative of the time-varying freestream Mach numbers associated with a helicopter rotor blade during forward flight, in which the oscillatory frequency $\omega_{M}$ corresponds to one period per revolution of the blade (i.e., 1/rev) [1] . For a typical rotor blade depicted in Fig. 2, with rotational velocity $\Omega$ and radius $R$, the Mach number at the blade tip in hover (i.e., zero forward flight velocity) is given by

$$
M_{\Omega R}=\frac{\Omega R}{a}
$$

The mean value and oscillatory amplitude in Eq. (ㄱ) are

$$
M_{0}=r M_{\Omega R}
$$

and

$$
\bar{M}=\mu M_{\Omega R}
$$

where $\mu$ is referred to as the advance ratio and is defined as the component of the forward flight velocity parallel to the hub plane of the rotor normalized by $\Omega R$.

The oscillatory frequencies in Eqs. (므그) are given by

$$
\omega_{\theta}=\frac{k_{\theta} V_{0}}{b}, \quad \omega_{h}=\frac{k_{h} V_{0}}{b}, \quad \omega_{M}=\frac{k_{M} V_{0}}{b}
$$

where

$$
V_{0}=a M_{0}
$$

Furthermore,

$$
\omega_{M}=\Omega
$$

which gives

$$
k_{M}=\frac{\Omega b}{a M_{0}}=\frac{\Omega b}{a r M_{\Omega R}}=\frac{b}{R r}
$$

Note that the unsteady aerodynamic loading is typically only modeled for $r>0.15$ in helicopter applications, since the loads near the hub are relatively insignificant and their effects on the aeroelastic response of the blade can be neglected. Therefore, for given values of the nondimensional airfoil semichord $b / R$ and $M_{\Omega R}$, a single training case given by Eqs. (5-7) is defined by six independent parameters: $\left(\bar{\theta}, k_{\theta}, \bar{h}, k_{h}, r, \mu\right)$.

DOE methods $[16,17]$ are used to select $N_{T}$ initial training cases for which to conduct CFD simulations. Each training case in the DOE consists of different combinations of the six independent parameters. In general, the number of training cases required to accurately identify a system will increase as the number of external inputs increases. For strongly nonlinear systems, additional external inputs can result in significant increases in the number of required training

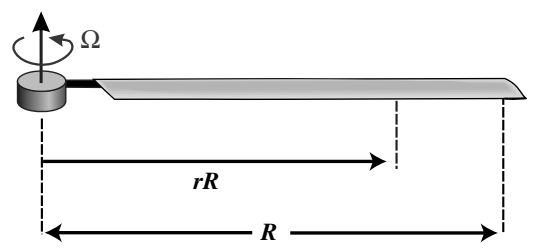

Fig. 2 Typical rotor blade configuration. 
cases (i.e., the curse of dimensionality). So, as with any reducedorder modeling method, the cost associated with the initial training cases may be prohibitive for applications involving numerous structural degrees of freedom. However, modal decomposition techniques can be useful for modeling structures with a tractable number of degrees of freedom.

When the initial data set is produced by a deterministic computer code, as opposed to a physical experiment or stochastic analysis, a given input will always yield the same output, because there is no measurement error or other random sources of noise. Under these conditions, the DOE need only be space-filling [26,29] so that all regions of the input parameter space are sampled. A commonly used space-filling design is Latin hypercube sampling (LHS) [30]. In LHS, each input parameter is partitioned into $N_{T}$ equally spaced sections. Each input parameter is sampled once in each section, resulting in a column vector containing $N_{T}$ different values of the input parameter. The column vectors for each input parameter are arranged side by side into a matrix and the components of the vectors are then randomly reordered. The resulting matrix is known as a Latin hypercube. Since there are six independent parameters in this study, each hypercube is a $N_{T} \times 6$ matrix, in which each row corresponds to a different training case defined by the six input parameters. A major disadvantage of LHS is that training cases can cluster together due to the random combination of input parameters associated with each row of the Latin hypercube. To prevent this, optimal Latin hypercube (OLH) sampling is used in this study to ensure a more uniform (or space-filling) design of experiment. Optimal Latin hypercube sampling creates a more uniform design than conventional LHS by maximizing a spreading criteria, rather than randomly combining input parameters. Figure 3 illustrates the difference between a conventional Latin hypercube and an optimal Latin hypercube for a twodimensional parameter space. In this study, the OLH algorithm from the iSIGHT software package was used [31-33].

\section{Extract Time-Domain Sample Data}

For each training case, CFD is used to obtain time-domain aerodynamic response data from $t_{0}$ to $t_{f}$, in increments of $\Delta t$. The sampled outputs and the corresponding inputs required to construct the surrogate mapping function $\hat{\Phi}$ are obtained from the CFD results. In this study, sample data from each training case is obtained for one period corresponding to the lowest-frequency component: i.e.,

$$
t_{f}-t_{0}=\frac{2 \pi}{\min \left[\omega_{\theta}, \omega_{h}, \omega_{M}\right]}
$$

From Eq. (4), the vector of inputs corresponding to a sampled output $y$ is given by

$$
\begin{gathered}
\mathbf{x}(t)=\left[\begin{array}{lll}
\mathbf{u}_{j}(t) \quad \mathbf{u}_{j}(t-\Delta t) \cdots \mathbf{u}_{j}(t-m \Delta t) & y_{j}(t-\Delta t) \cdots y_{j}(t-n \Delta t
\end{array}\right] \\
\text { for } j=1,2, \ldots, N_{T}
\end{gathered}
$$

where $y_{j}$ and $\mathbf{u}_{j}$ correspond to the $j$ th training case and are given by Eqs. (2) and (3), and $\mathbf{x}$ is a vector of size $N_{x}=7(m+1)+n$, where the factor of 7 is due to the size of the external input vector given by Eq. (3).
For every training case in the OLH, fitting data of the form $(\mathbf{x}, y)$ is available at discrete time instants $t$, for $\left(t_{0}+\max [m, n] \Delta t\right) \leq t \leq t_{f}$. At time $t$ of the $j$ th training case, $\mathbf{u}_{j}(t)$ and $\mathbf{u}_{j}(t-\Delta t), \ldots, \mathbf{u}_{j}(t-$ $m \Delta t$ ) are known from the prescribed motions defined by Eqs. (5-7), and the responses $y_{j}(t)$ and $y_{j}(t-\Delta t), \ldots, y_{j}(t-n \Delta t)$ are obtained from the CFD results. The sample data obtained from each of the $N_{T}$ training cases are combined into a single data set consisting of $N_{\mathrm{sp}}$ total sample points: i.e., $\left(\mathbf{x}^{(i)}, y^{(i)}\right)$ for $i=1,2, \ldots, N_{\text {sp. }}$. Note that generating the sample data can be expedited by using parallel computation, since multiple training cases in the OLH can be simulated simultaneously.

\section{Construct Surrogate Mapping Function (Kriging Interpolation)}

The next step in constructing the reduced-order aerodynamic model is to approximate the nonlinear mapping function by interpolating the sampled data. The resulting surrogate mapping function $\hat{\Phi}$ is used in place of the exact function in order to generate approximate predictions of the unsteady airloads for arbitrary inputs. To be useful for modeling nonlinear flow effects, the interpolation method must be well suited to approximating nonlinear functions and should not require a priori assumptions on the form of the function that is to be approximated. Kriging interpolation [27,28] satisfies these conditions and is therefore used to generate the surrogate mapping functions in this study.

In kriging, the unknown function of interest, $\Phi(\mathbf{x})$, is assumed to be a random variable of the form

$$
\Phi(\mathbf{x})=f(\mathbf{x})+Z(\mathbf{x})
$$

where $f(\mathbf{x})$ is an assumed function (usually a low-order polynomial) and $Z(\mathbf{x})$ is a stochastic (random) process that is assumed to be Gaussian with zero mean and variance $\sigma_{\mathrm{var}}^{2}$. The regression model $f(\mathbf{x})$ can be thought of as a globally valid trend function, while $Z(\mathbf{x})$ accounts for local deviations from $f(\mathbf{x})$ that ensure that the kriging model interpolates the sample points exactly. The local deviations dictated by the sample points enable the kriging predictor to approximate nonlinear behavior regardless of the exact function's form. Note that although $\Phi(\mathbf{x})$ is deterministic rather than the stochastic process assumed in Eq. (17), kriging interpolation is still applicable. The assumption that $\Phi \overline{(\mathbf{x})}$ is a random process is made because the deviation from the regression model can resemble a realization of a stochastic process [27].

The covariance matrix of $Z(\mathbf{x})$, which is a measure of how strongly correlated two points are, is given by

$$
\operatorname{Cov}\left[Z\left(\mathbf{x}^{(i)}\right), Z\left(\mathbf{x}^{(j)}\right)\right]=\sigma_{\mathrm{var}}^{2} \mathbf{R}_{\mathrm{krg}}
$$

where each element of the $N_{\mathrm{sp}} \times N_{\mathrm{sp}}$ correlation matrix $\mathbf{R}_{\mathrm{krg}}$ is given by

$$
\left(R_{\mathrm{krg}}\right)_{i j}=R_{\mathrm{krg}}\left(\mathbf{x}^{(i)}, \mathbf{x}^{(j)}\right)
$$

and $R_{\mathrm{krg}}$ is a user defined spatial correlation function (SCF). In this study,

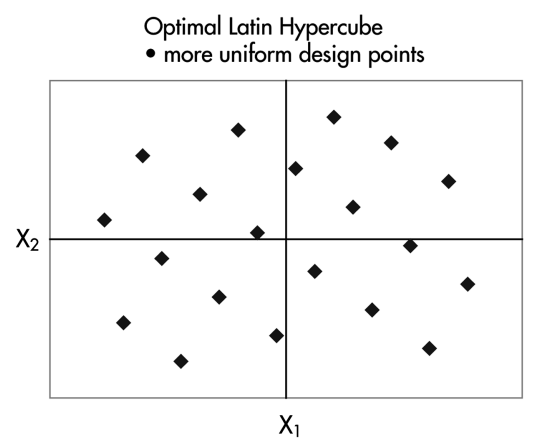

Fig. 3 Conventional Latin hypercube vs optimal Latin hypercube in two-dimensional parameter space. 


$$
R_{\mathrm{krg}}\left(\mathbf{x}^{(i)}, \mathbf{x}^{(j)}\right)=\prod_{k=1}^{N_{x}} \max \left[0,1-p_{k}\left|x_{k}^{(i)}-x_{k}^{(j)}\right|\right]
$$

where $x_{k}^{(i)}$ is the $k$ th component of the $i$ th sample input given by Eq. (16). This SCF was selected because the resulting correlation matrices were not ill-conditioned, which is an issue that may arise with kriging [28]. As two points move closer to each other, $\mid x_{k}^{(i)}-$ $x_{k}^{(j)} \mid \rightarrow 0$, and Eq. (20) approaches unity, which is the maximum value of the SCF. Therefore, the SCF recovers the intuitive property that the closer two points are to each other, the greater the correlation between the points.

The fitting parameters $p_{k}$ are unknown correlation parameters that need to be determined. To determine these parameters, the form of $f(\mathbf{x})$ needs to be chosen. In this study, $f(\mathbf{x})$ is assumed to be a second-order polynomial given by

$$
f(\mathbf{x})=f_{x}^{T} \boldsymbol{\beta}
$$

where $f_{x}^{T}$ is a $1 \times N_{\text {basis }}$ vector, $N_{\text {basis }}$ is the number of basis functions associated with the second-order polynomial, and $\beta$ is a $N_{\text {basis }} \times 1$ vector of coefficients. Similarly, $\mathbf{F}(\mathbf{x})$ can be defined as an $N_{\mathrm{sp}} \times$ $N_{\text {basis }}$ matrix, where the $i$ th row corresponds to the evaluation of the $N_{\text {basis }}$ functions at the $i$ sample point.

To find $p_{k}$, the generalized least-squares estimates of $\boldsymbol{\beta}$ and $\sigma_{\mathrm{var}}^{2}$, denoted by $\hat{\boldsymbol{\beta}}$ and $\hat{\sigma}_{\text {var }}^{2}$, respectively, are employed [27,28]:

$$
\hat{\boldsymbol{\beta}}=\left(\mathbf{F}^{T}\left(\mathbf{R}_{\mathrm{krg}}\right)^{-1} \mathbf{F}\right)^{-1} \mathbf{F}^{T}\left(\mathbf{R}_{\mathrm{krg}}\right)^{-1} \mathbf{y}
$$

and

$$
\hat{\sigma}_{\mathrm{var}}^{2}=\frac{(\mathbf{y}-\mathbf{F} \hat{\boldsymbol{\beta}})^{T}\left(\mathbf{R}_{\mathrm{krg}}\right)^{-1}(\mathbf{y}-\mathbf{F} \hat{\boldsymbol{\beta}})}{N_{\mathrm{sp}}}
$$

where $\mathbf{y}$ is a $N_{\mathrm{sp}} \times 1$ vector of observed function outputs at the sampled inputs.

With $\hat{\sigma}_{\text {var }}^{2}$ and $\hat{\boldsymbol{\beta}}$ known, $p_{k}$ are found such that a likelihood function is maximized [28,34]. The likelihood function, given in Eq. (24), represents the probability that the stochastic process $\Phi(\mathbf{x})$ produced the sampled data points in $\mathbf{y}$. Since the stochastic process associated with kriging has been assumed to be Gaussian, one seeks the set $p_{k}$ that maximizes the probability that the sample points have been drawn from a Gaussian process:

$$
L\left(\mathbf{y}, p_{k}\right)=-\frac{N_{\mathrm{sp}} \ln \left(\hat{\sigma}_{\mathrm{var}}^{2}\right)+\ln \left|\mathbf{R}_{\mathrm{krg}}\right|}{2}
$$

The maximum likelihood estimates of $p_{k}$ represent the fitting parameters that are most consistent with the sampled data. Any values of $p_{k}$ would result in a surrogate that interpolates the sample points exactly, but the "best" kriging surrogate is found by maximizing the likelihood function.

With all parameters known, the kriging approximation of a function $\Phi(\mathbf{x})$ is given by [27]

$$
\hat{\Phi}(\mathbf{x})=f_{x}^{T} \hat{\boldsymbol{\beta}}+\mathbf{r}_{\mathrm{krg}}^{T}(\mathbf{x})\left(\mathbf{R}_{\mathrm{krg}}\right)^{-1}(\mathbf{y}-\mathbf{F} \hat{\boldsymbol{\beta}})
$$

where

$$
\mathbf{r}_{\mathrm{krg}}(\mathbf{x})=\left[R_{\mathrm{krg}}\left(\mathbf{x}, \mathbf{x}^{(1)}\right), R_{\mathrm{krg}}\left(\mathbf{x}, \mathbf{x}^{(2)}\right), \ldots, R_{\mathrm{krg}}\left(\mathbf{x}, \mathbf{x}^{\left(N_{\mathrm{sp}}\right)}\right)\right]^{T}
$$

The column vector $\mathbf{r}_{\mathrm{krg}}(\mathbf{x})$ of length $N_{\mathrm{sp}}$ is the correlation vector between an arbitrary prediction point $\mathbf{x}$ and the sampled inputs, $\mathbf{x}^{(1)}, \ldots, \mathbf{x}^{\left(N_{s p}\right)}$. The kriging predictor given by Eq. (25) represents the optimal predictor in the sense that, among all admissible predictors, Eq. (25) results in the minimum mean square error with respect to the assumed stochastic process $\Phi(\mathbf{x})$ [27]. Note that although the kriging predictor is derived as an approximation to a stochastic process, Eq. (25) is a deterministic function. Therefore, kriging has been used extensively in approximating deterministic computer models [28]. The kriging surrogates were created with a freely available MATLAB toolbox [35].
It is worth noting that several design of experiment (DOE) and nonlinear interpolation methods have been implemented in popular engineering software packages such as MATLAB. For example, a kriging surrogate for the mapping function could be generated with MATLAB by first using the lhsdesign function in order to produce an optimal Latin hypercube for the DOE. A CFD solver would then be used to obtain the time-domain aerodynamic response data corresponding to the cases in the Latin hypercube. Finally, the full-order input/output data would be provided as inputs to the MATLAB kriging toolbox (see [35]), which would automatically generate the surrogate. Furthermore, users are not limited to kriging surrogates based on optimal Latin hypercubes, since alternative DOE methods are available in the MATLAB Statistics Toolbox, and additional nonlinear interpolation methods can be found in the System Identification and Neural Network Toolboxes. Therefore, the reduced-order modeling approach described in this paper can be setup with relatively little difficulty, since well-documented DOE and nonlinear interpolation tools are widely available.

\section{Time-Domain Predictions Using a Surrogate-Based \\ Recurrence Framework}

The surrogate mapping function can be used for discrete timedomain predictions when provided with arbitrary instances of the input vector $\mathbf{x}(t)$ given by Eq. (16). However, $n$ components of $\mathbf{x}$ correspond to previous values of the unsteady aerodynamic response, which will not be known for arbitrary inputs. Only the surrogate's approximation of the previous responses will be available. Therefore, an approximate input vector $\hat{\mathbf{x}}$ consisting of the surrogate's predictions of the previous responses must be used in order to obtain predictions at an arbitrary input. The approximate input vector is

$$
\hat{\mathbf{x}}(t)=\left[\begin{array}{lll}
\mathbf{u}(t) & \mathbf{u}(t-\Delta t) \cdots \mathbf{u}(t-m \Delta t) & \hat{y}(t-\Delta t) \cdots \hat{y}(t-n \Delta t)
\end{array}\right]
$$

where the approximate previous responses $\hat{y}$ are obtained from the surrogate's predictions at previous time steps. The development of the reduced-order aerodynamic model is completed by using the surrogate mapping function within the recurrence framework depicted in Fig. 4 . The term recurrence refers to the property that the approximate solutions, $\hat{y}$, are fed back and used as inputs to $\hat{\Phi}$ for the prediction at the current time. Therefore, the approximate aerodynamic responses obtained from the surrogate-based recurrence framework (SBRF) can be written as

$$
\hat{y}(t)=\hat{\Phi}(\hat{\mathbf{x}}(t))
$$

The process depicted in Fig. 4 is marched forward in increments of $\Delta t$ until the approximate solution at some final time is obtained. Note that guesses for the $n$ previous responses are needed to initialize the SBRF. However, as will be shown in Sec. IV, the accuracy of the

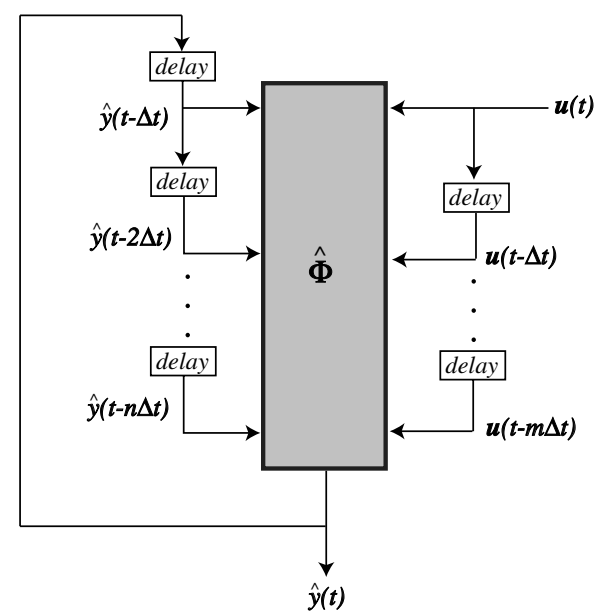

Fig. 4 Surrogate-based recurrence framework. 
initial guesses is not critical, since the initial transients in the SBRF's predictions die out as time is marched forward. This is similar to CFD solutions, which must use a number of initial time steps to allow transients in the solution to settle. In summary, the SBRF accounts for time-history effects associated with unsteady flows by employing a recurrence solution methodology, while flow nonlinearities are modeled by using a kriging approximation of the full-order mapping function.

\section{CFD Solver}

The CFD results generated in this study are obtained using the commercially available CFD code CFD++ $[36,37]$ developed by Metacomp Technologies. The CFD++ code is capable of solving the compressible unsteady Reynolds-averaged Navier-Stokes equations. It uses a unified grid methodology that can handle a variety of structured, unstructured, multiblock meshes, and cell types, including patched and overset grid features. Spatial discretization of the Navier-Stokes equations is based on a second-order multidimensional total-variation-diminishing scheme [38]. For temporal discretization an implicit algorithm with dual-time-stepping and multigrid acceleration is used. Dual-time-stepping schemes are constructed by appending a pseudo-time-derivative term and using subiterations for improved accuracy. Multigrid acceleration employs a hierarchy of coarsening grids to speed the convergence. Several turbulence models are available in CFD++, ranging from oneequation to three-equation transport models.

All the computations are carried out by solving RANS equations and assuming fully turbulent flow. The computational domain is a C-grid, as shown in Fig. 5, with far-field boundary extending to 50 chord lengths in all directions. A distance of 30-50 chord lengths is typically used in order to minimize the effects of numerical reflection from the far-field boundary. The grids are clustered at the airfoil wall boundaries such that the dimensionless distance $y^{+}$of the first grid point off the wall is less than 1 and the equations are directly solved to the walls without assuming any wall functions. The SpalartAllmaras turbulence model is used in all computations.

\section{Results}

The effectiveness of the SBRF approach is demonstrated in this section by comparing against full-order CFD predictions for unsteady lift, moment, and drag coefficients for a NACA 0012 airfoil subject to fixed and time-varying freestream Mach numbers. The CFD data was generated for three periods of the lowest-frequency component so that initial transients in the solutions were eliminated. The data from the final period was used for training and testing the SBRF. For a time step of $\Delta t=0.0002 \mathrm{~s}$, the CFD results corresponding to each training/test case required $1-4 \mathrm{~h}$ of simulation time using four $3.2 \mathrm{GHz}$ Xeon processors. The values of $m$ and $n$ were selected after trying various combinations of the parameters with values up to 4 . For the flow regimes considered in this study, setting

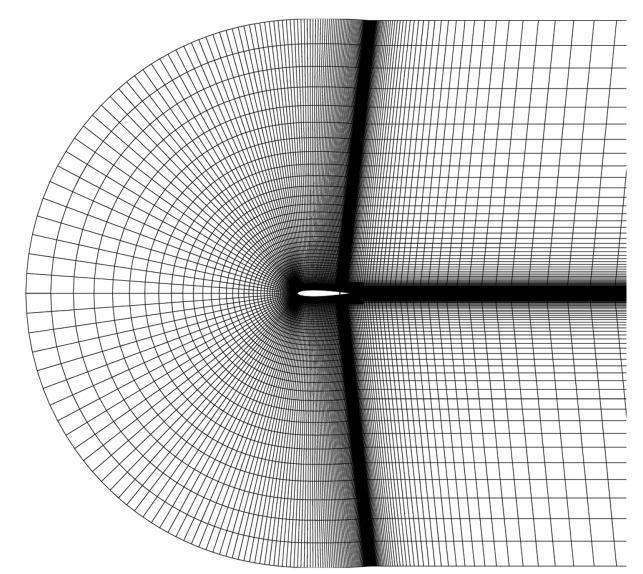

Fig. 5 C-grid used in CFD calculations. $m=0$ and $n=2$ resulted in a tractable number of inputs to the surrogate mapping function while enabling accurate approximation of time-history effects. Since increasing $m$ or $n$ did not result in significant improvement in the accuracy of the SBRF, all SBRF results presented in this study correspond to settings of $m=0$ and $n=2$.

The following error metric was used to quantify the accuracy of the SBRF approach relative to CFD:

$$
E^{(j)}=100 \times \frac{\frac{1}{N_{t}} \sum_{i=1}^{N_{t}}\left|\hat{y}\left(t_{i}\right)-y\left(t_{i}\right)\right|}{y_{\max }^{(j)}-y_{\min }^{(j)}}
$$

In Eq. (29), $E^{(j)}$ is a relative error measure corresponding to the $j$ th test case, the numerator corresponds to the average error over the total number of time steps, and the denominator represents the range of the exact response. Thus, Eq. (29) represents the average error in the SBRF relative to the range of the exact response.

To initialize the SBRF, responses corresponding to $n=2$ previous time steps are required as inputs to the surrogate mapping function. These initial responses were obtained from second-order polynomial response surfaces [17] that were only a function of the external inputs (i.e., no previous response inputs). Therefore, the predictions of the previous response quantities required to initialize the SBRF are given by

$$
\hat{y}(t-i \Delta t)=\hat{\Phi}_{\mathrm{qs}}(\mathbf{u}(t-i \Delta t)) \quad \text { for } \quad i=1, \ldots, n
$$

where $\hat{\Phi}_{\mathrm{qs}}$ denotes that these response surfaces can be thought of as quasi-steady approximations, since they are only functions of the instantaneous external inputs and therefore have no mechanism to account for time-history effects. After the initial $n$ time steps, the previous responses required as inputs are obtained from the SBRF feedback mechanism depicted in Fig. 4 .

\section{A. Pitch Motion and Fixed Freestream Mach Number}

The purpose of considering a pitching airfoil at a transonic Mach number is so that the effectiveness of the SBRF approach can be compared to a popular alternative reduced-order modeling approach described in the literature. The results presented in this section correspond to a modeling problem similar to the one considered in [39], in which a POD-based approach was used to model the unsteady lift of an NLR 7301 airfoil oscillating at a transonic Mach number $M=0.764$. The POD model was constructed from CFD solutions corresponding to 450 different combinations of $\bar{\theta}$ and $k_{\theta}$, which were constrained to be

$$
\begin{gathered}
0.0001^{\circ} \leq \bar{\theta} \leq 14^{\circ} \\
0.0 \leq k_{\theta} \leq 1.2
\end{gathered}
$$

The POD model based on 450 training cases was then used to predict unsteady lift coefficients corresponding to reduced frequencies of 0.2, 0.6, and 1.2. Overall, the POD model predictions compared well with CFD, although there were noticeable errors (around 10\%) for certain cases.

In this study, a SBRF constructed from only 200 training cases for a NACA 0012 airfoil at $M=0.764$ was considered. The 200 point OLH used to generate the training data was constrained by Eqs. (31) and (32). The SBRF predictions of unsteady lift were compared to CFD results corresponding to 18 test cases similar to those considered in [39]. The test cases, which were not included in the 200 point OLH, corresponded to $\bar{\theta}=2,4,6,8,10$, and $12^{\circ}$ at each of the three reduced frequencies $k_{\theta}=0.2,0.6$, and 1.0. According to the metric defined in Eq. (29), the SBRF errors ranged from 0.2-2.8\%, with an average value of $1.3 \%$ over the 18 test cases. Comparisons of the SBRF predictions and CFD are shown in Fig. $\underline{6}$ for six of the 18 test cases. These results are representative of the accuracy exhibited by the SBRF predictions for all 18 test cases. Note that the case shown in Fig. 6a corresponds to the maximum error of $2.8 \%$. Therefore, even for the worst case, the SBRF based on 200 training 


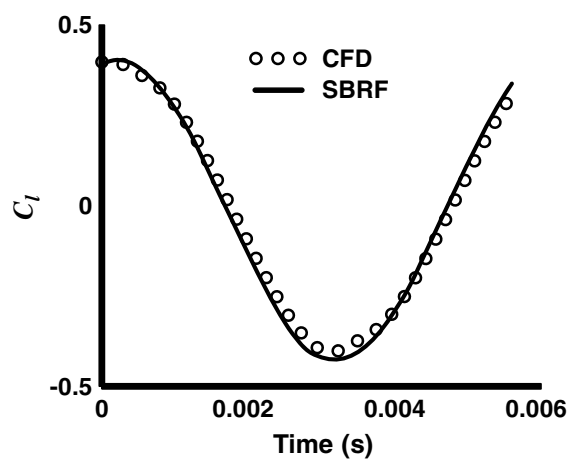

a) $\bar{\theta}=4^{\circ}, k=0.2$

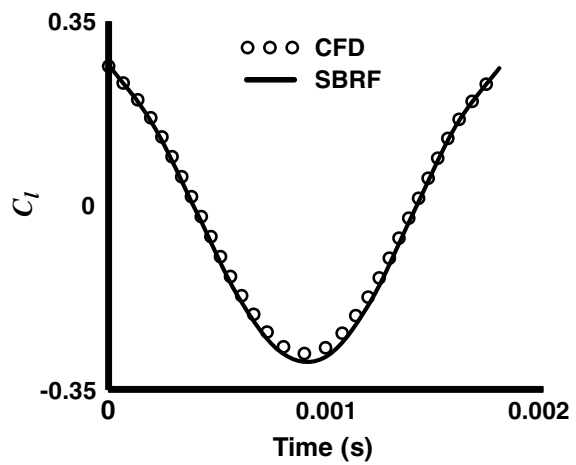

c) $\bar{\theta}=4^{\mathrm{o}}, k=0.6$

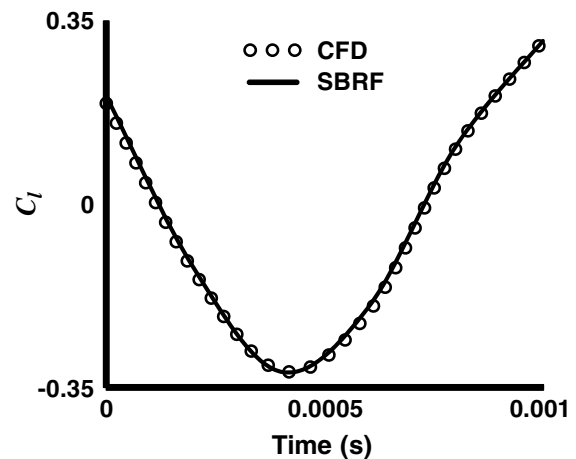

e) $\bar{\theta}=4^{\circ}, k=1.0$

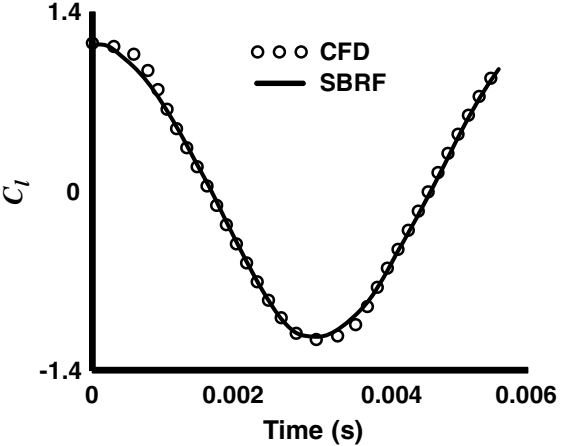

b) $\bar{\theta}=12^{\circ}, k=0.2$

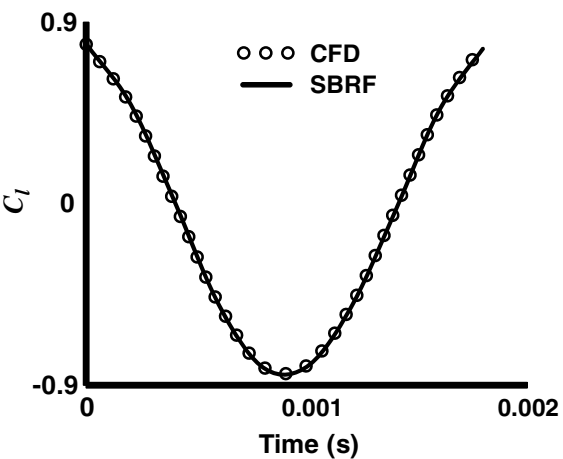

d) $\bar{\theta}=12^{\circ}, k=0.6$

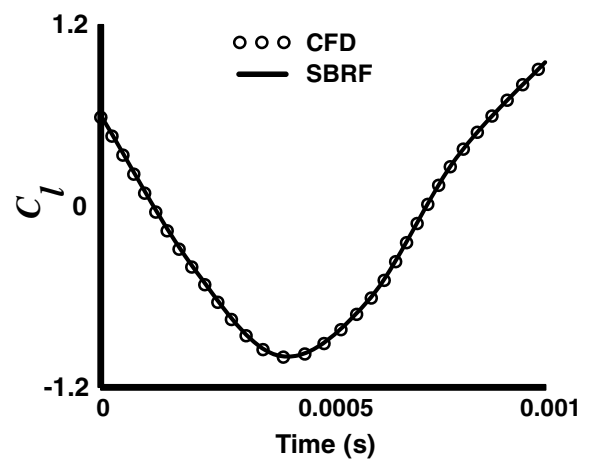

f) $\bar{\theta}=12^{\circ}, k=1.0$

Fig. 6 Unsteady lift coefficients for a pitching airfoil at $M=0.764$.

cases effectively mimics the CFD predictions of unsteady lift. Considering that 450 training cases were used in [39] for the POD approach, these results indicate that the SBRF approach compares favorably with alternative reduced-order modeling methods.

\section{B. Pitch/Plunge Motion and Fixed Freestream Mach Number}

To isolate the effectiveness of the SBRF in modeling aerodynamic nonlinearities, the SBRF was compared with CFD and the linear RFA approach [2] for a fixed freestream Mach number. The RFA approach is a 2-D unsteady time-domain theory that accounts for compressibility as well as variations in the oncoming flow velocity, and is based on Roger's approximation [40] for representing aerodynamic loads in the Laplace domain. In the RFA implementation [2], the oscillatory airloads in the frequency domain are obtained from a 2-D doublet-lattice solution [41] of Possio's integral equation [23]. The Laplace domain transfer matrix that maps generalized airfoil motions into generalized aerodynamic loads is obtained from a least-squares fit of the frequency domain data. A time-domain representation of the aerodynamic transfer matrix is obtained by taking the inverse Laplace. The Mach number $M=0.75$ was chosen so that flow nonlinearities due to transonic effects would be present. The accuracy of the SBRF and RFA predictions was quantified by comparing with CFD results for a set of test cases that were not included in the OLH used to train the surrogate mapping function. Since the Mach number is fixed, only four parameters are required to define a training case: $\bar{\theta}, k_{\theta}, \bar{h}$, and $k_{h}$. Furthermore, the surrogate mapping function will be a function of seven inputs: $\theta(t)$, $\dot{\theta}(t), \ddot{\theta}(t), \dot{h}(t), \ddot{h}(t), \hat{y}(t-\Delta t)$, and $\hat{y}(t-2 \Delta t)$. An OLH consisting of 150 pitch/plunge motions was used to generate the sample points, while test points were generated from a separate 20-point OLH. The bounds on the OLH parameters were

$$
\begin{gathered}
0^{\circ} \leq \bar{\theta} \leq 8^{\circ} \\
0.01 \leq k_{\theta} \leq 0.3 \\
0 \mathrm{~m} \leq \bar{h} \leq 0.023 \mathrm{~m} \\
0.01 \leq k_{h} \leq 0.3
\end{gathered}
$$

The upper bound for $\bar{h}$ was selected so that

$$
\dot{h}_{\max }=V_{0}\left(\theta_{\max } \pi / 180\right)
$$


Table 1 Comparison of SBRF and RFA errors for 20 test cases $(M=0.75)$

\begin{tabular}{lccc}
\hline \hline Method & Error in $C_{l}$ & Error in $C_{m}$ & Error in $C_{d}$ \\
\hline SBRF & $0.3-1.9 \%($ avg $0.7 \%)$ & $2.4-8.0 \%($ avg $4.7 \%)$ & $1.5-10.3 \%($ avg $3.9 \%)$ \\
RFA & $2.7-11.1 \%($ avg $6.6 \%)$ & $5.0-35.1 \%($ avg $15.5 \%)$ & - \\
\hline \hline
\end{tabular}

where $\dot{h}_{\max }$ and $\theta_{\max }$ represent the maximum possible plunge velocity and pitch angle, respectively.

A comparison of the SBRF and RFA errors for the 20 test cases is presented in Table 1 . Note that the RFA approach does not calculate drag, so a corresponding error measure is not available. The SBRF and RFA approaches required a fraction of a second on a single computer processor to generate the predictions for each test case, while the CFD results required $1-4 \mathrm{~h}$ to generate using four processors. The average errors over all 20 test cases for the SBRF were $0.7,4.7$, and $3.9 \%$ for lift, moment, and drag, respectively. Similarly, the average RFA errors were 6.6 and $15.5 \%$ for lift and moment.

The advantage of the nonlinear reduced-order model is best illustrated by the unsteady moment predictions: the SBRF corresponds to $2.4-8.0 \%$ error for the 20 test cases, while the RFA approach results in $5.0-35.1 \%$ error. The improved predictions facilitated by the SBRF are expected, since the moment coefficient is very sensitive to the position of shocks that move over the airfoil's surface in transonic flow. The linear RFA approach cannot model such flow nonlinearities. Similarly, it was noted in [3] that the indicial function approach also has difficulty modeling nonlinear transonic shock effects on the unsteady moment. In contrast, the nonlinear SBRF approach accurately modeled transonic effects for the 20 test cases. This is illustrated in Fig. 7, which shows the unsteady airloads predicted by CFD, the SBRF, and RFA (lift and moment) for one of the 20 test cases that demonstrated a significant effect on the moment coefficient due to a moving shock. For this test case, the SBRF errors are $0.4,4.1$, and $2.1 \%$ for lift, moment, and drag, respectively, while the RFA approach corresponds to 3.6 and $21.8 \%$ error for lift and moment. The results shown in Fig. 7 demonstrate the ability of the
SBRF approach to mimic CFD solutions for highly nonlinear flows. However, the SBRF predictions required a fraction of a second, while the CFD results for this test case required $3 \mathrm{~h}$ to generate. Therefore, the SBRF can accurately approximate the fidelity associated with CFD, while exhibiting the computational efficiency associated with approximate linear models.

To investigate the effects of how the SBRF is initialized, the SBRF initialized with predictions from the quasi-steady response surface was compared with an SBRF initialized by setting $\hat{y}(t-i \Delta t)=0$ for $i=1, \ldots, n$. The latter case is representative of the SBRF's performance when initializing with poor guesses for the previous responses. The effects of the poor initial guesses were found to be negligible for the 20 test cases, since they only affected the SBRF's predictions in the vicinity of the initial time. As time was marched forward, the initial transients died out and the SBRF's predictions quickly reached a periodic solution. This is illustrated in Fig. $\underline{8}$, which corresponds to the test case depicted in Fig. 7. In Fig. $\underline{8}$, SBRF_QS denotes initial quantities obtained from the quasi-steady response surface, while SBRF_0 corresponds to initial responses set to zero. It is clear from Fig. 8 that the SBRF settles into a periodic solution for both methods of initialization. Therefore, the method for estimating the initial responses is not critical.

In the preceding results, the SBRF was trained and tested by considering simple harmonic motions. However, in practice, a timedomain aerodynamic model must accurately predict unsteady aerodynamic responses for arbitrary combinations of the components of the input vector $\mathbf{x}$. To demonstrate the effectiveness of the SBRF for aerodynamic response predictions due to arbitrary motion, a multiharmonic pitching motion given by Eq. (하) was considered:

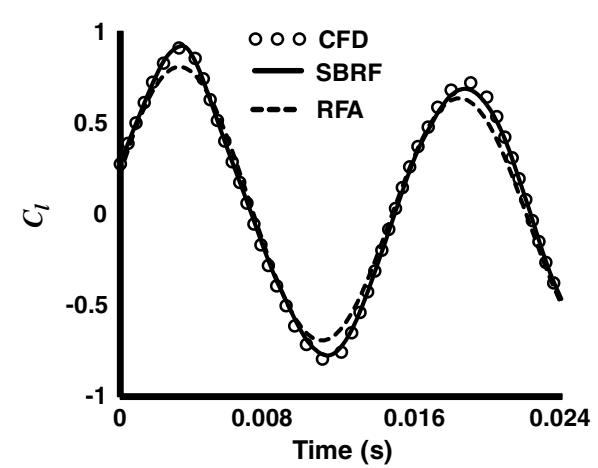

a) Lift coefficient

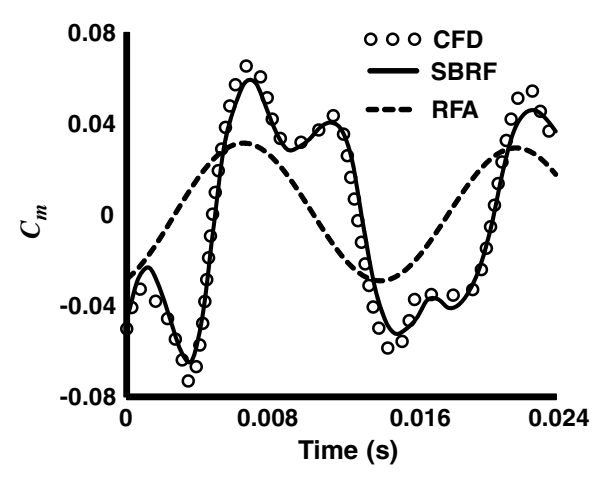

b) Moment coefficient

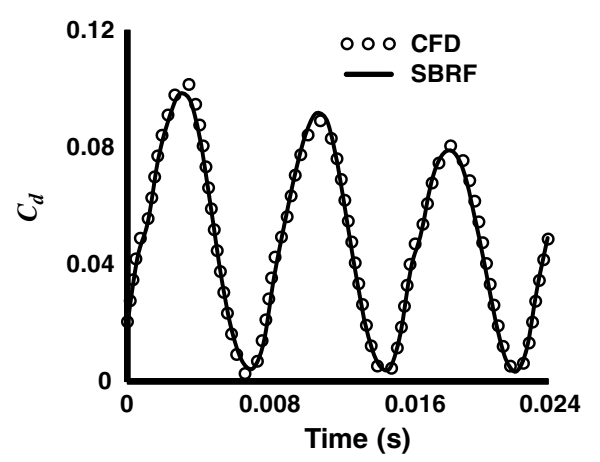

c) Drag coefficient

Fig. 7 Unsteady lift, moment, and drag coefficients $\left(\bar{\theta}=5.9^{\circ}, k_{\theta}=0.08, \bar{h}=0.013 \mathrm{~m}, k_{h}=0.05\right.$, and $\left.M=0.75\right)$. 


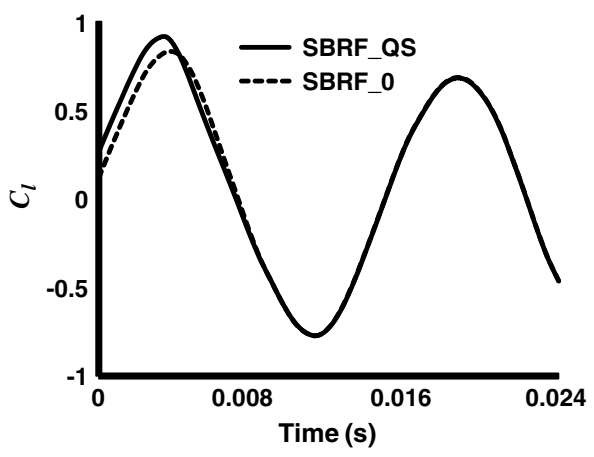

a) Lift coefficient

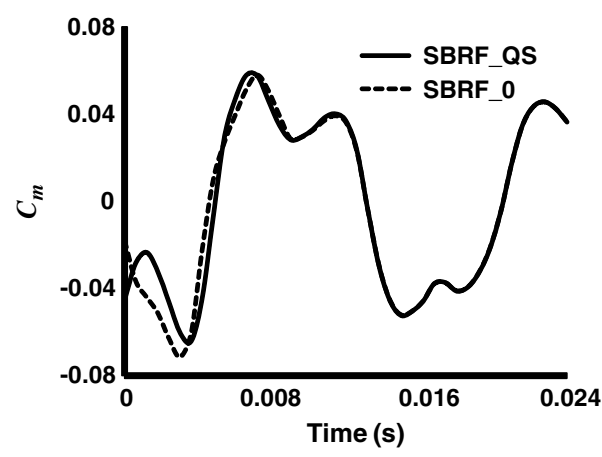

b) Moment coefficient

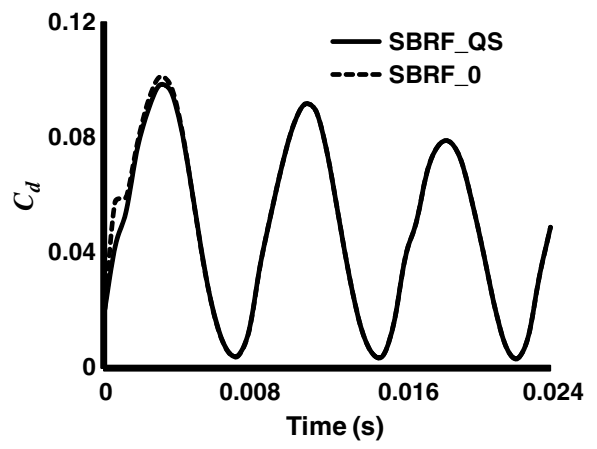

c) Drag coefficient

Fig. 8 Comparison of SBRF predictions with different initialization methods $\left(\bar{\theta}=5.9^{\circ}, k_{\theta}=0.08, \bar{h}=0.013 \mathrm{~m}, k_{h}=0.05\right.$, and $\left.M=0.75\right)$.

$$
\theta(t)=\sum_{i=1}^{50} \bar{\theta}_{i} \cos \left(\omega_{\theta, i} t+\phi_{i}\right)
$$

The 50-component multiharmonic pitch motion was generated by randomly selecting the amplitudes $\bar{\theta}_{i}$, frequencies $\omega_{\theta, i}$, and phase shifts $\phi_{i}$. The randomly generated motion was constrained such that the resulting $\theta(t), \dot{\theta}(t)$, and $\ddot{\theta}(t)$ were within the bounds of the sample data so that extrapolation was avoided. As with any interpolation method, the SBRF's predictions are not reliable when used for extrapolation. The plunge degrees of freedom, $\dot{h}$ and $\ddot{h}$, were set to zero for this case. The randomly generated pitch input, and the comparison between the SBRF approach and CFD, are shown in Fig. 9. It is clear from Fig. 9 that the SBRF accurately predicts the
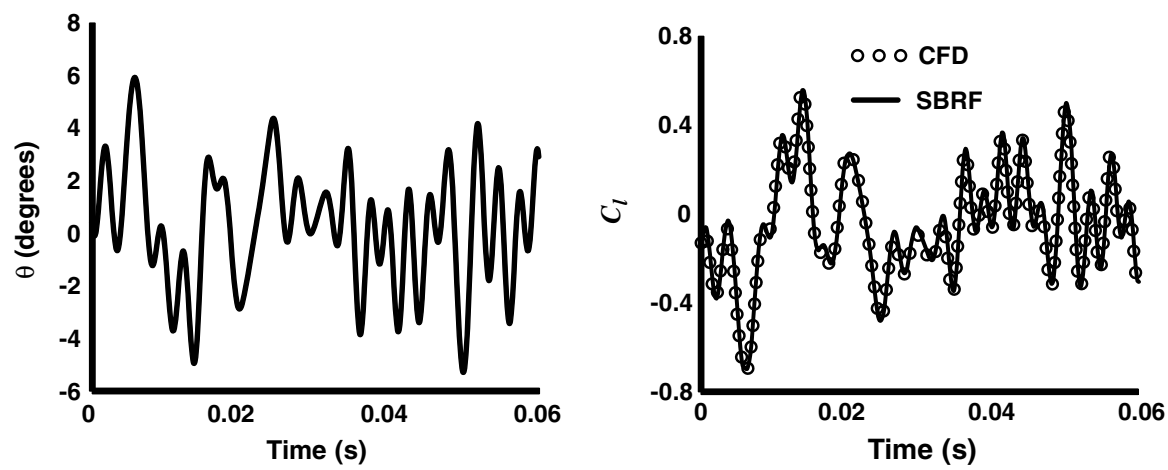

a) Pitch angle

b) Lift coefficient
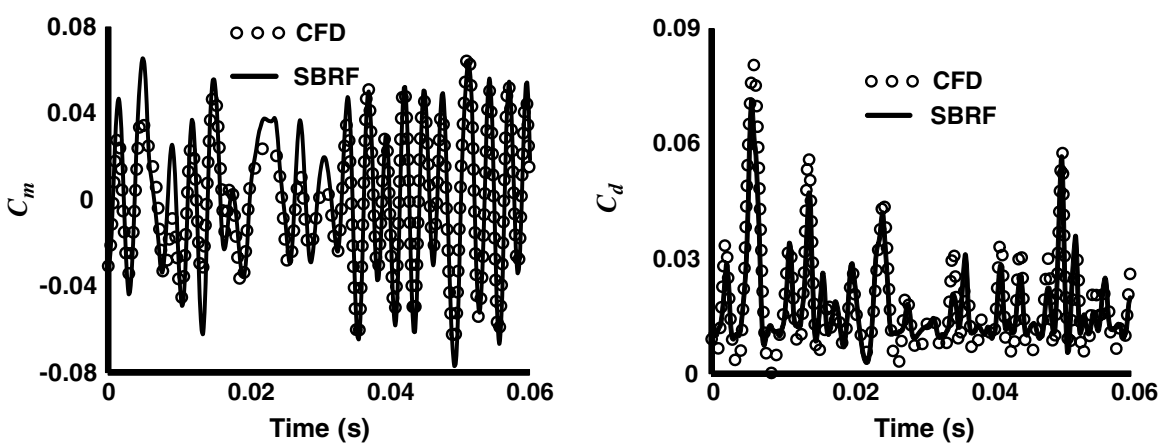

d) Drag coefficient

Fig. 9 Unsteady lift, moment, and drag coefficients for multiharmonic pitch input $(M=0.75)$. 
Table 2 Comparison of SBRF and RFA errors for 20 test cases subject to time-varying Mach numbers

\begin{tabular}{lccc}
\hline \hline Method & Error in $C_{l}$ & Error in $C_{m}$ & Error in $C_{d}$ \\
\hline SBRF & $1.0-4.5 \%($ avg $2.1 \%)$ & $1.6-7.9 \%($ avg $3.6 \%)$ & $2.0-10.0 \%($ avg $5.5 \%)$ \\
RFA & $0.6-5.4 \%($ avg 3.1\%) & $1.2-18.4 \%($ avg $6.1 \%)$ & - \\
\hline \hline
\end{tabular}

unsteady airloads corresponding to the multiharmonic motion. The SBRF errors are 1.2, 4.0, and 5.2\% for lift, moment, and drag, respectively. These results indicate that generating the training data from an adequate number of simple harmonic airfoil motions can produce a sufficiently space-filling set of sample points such that the SBRF has enough information from surrounding sample points to accurately interpolate at arbitrary inputs.

\section{Pitch/Plunge Motion and Time-Varying Freestream Mach Numbers}

To be applicable to rotary-wing applications, the SBRF approach must account for time-varying Mach numbers. The results in this section demonstrate the effectiveness of the SBRF approach for modeling unsteady aerodynamic responses subject to time-varying Mach numbers. All time-varying Mach number results correspond to $b / R=0.027$ and $M_{\Omega R}=0.64$. These parameters are consistent with a model representative of an MBB BO-105 helicopter rotor. When modeling time-varying Mach numbers, $r$ and $\mu$ must be included in the OLH in addition to the four parameters required for the fixed-Mach-number case. The surrogate mapping function requires $M(t)$ and $\dot{M}(t)$ as inputs in addition to the seven variables used in the fixed-Mach-number case. An OLH consisting of 200 training cases was used to generate the sample data, while test points were generated from a separate 20-point OLH. The bounds on the pitch/plunge kinematical parameters are the same as those given by Eqs. (33- $\underline{36})$, and

$$
\begin{gathered}
0.75 \leq r \leq 0.85 \\
0 \leq \mu \leq 0.4
\end{gathered}
$$

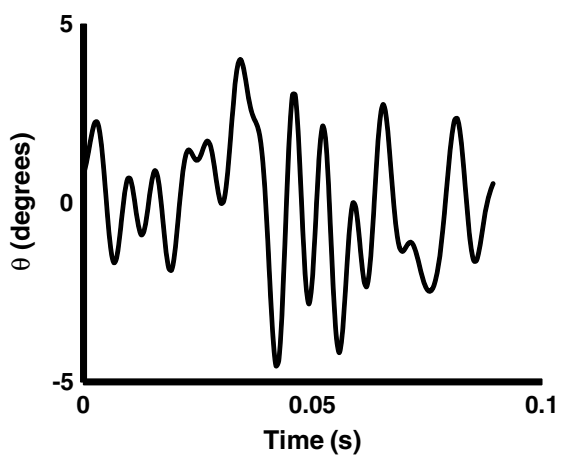

a) Pitch angle

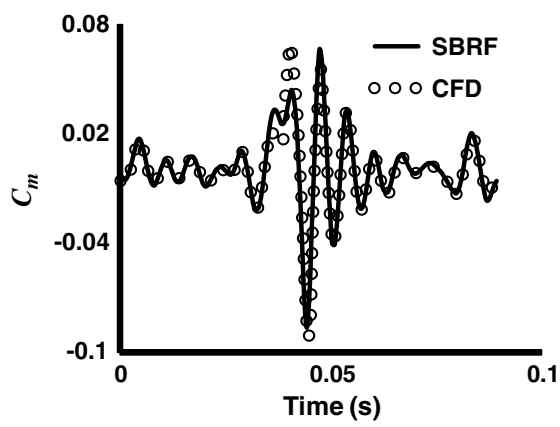

c) Moment coefficient
Therefore, the effectiveness of the SBRF for modeling time-varying Mach number effects is demonstrated in this study by considering a spanwise section spanning the $75-85 \%$ blade stations, over advance ratios that range from hover $(\mu=0)$ to high-speed forward flight.

The SBRF and RFA errors for the 20 test cases are presented in Table 2. For these cases, the differences between the SBRF and RFA errors were not as large as the fixed-Mach-number cases. This is because the time-varying test cases correspond to Mach numbers that vary from 0.24 to 0.79 . Therefore, the flow conditions will vary between linear conditions at the lower Mach numbers and nonlinear conditions at transonic Mach numbers. The RFA approach is expected to be more accurate for the low-Mach-number portions of the test cases. The largest difference between the SBRF and RFA predictions correspond to the maximum errors for moment coefficient: $7.9 \%$ for the SBRF and $18.4 \%$ for the RFA approach. This indicates that the SBRF is more accurate in the instances corresponding to strong transonic effects on the moment coefficient. Therefore, in addition to producing unsteady drag predictions, the advantage of the SBRF approach is that it accurately predicts for both linear and nonlinear flow conditions associated with time-varying Mach numbers.

As in Sec. IV.B, the effectiveness of the SBRF for modeling unsteady aerodynamic responses corresponding to arbitrary inputs (i.e., not limited to simple harmonic) was verified by considering a randomly generated pitch motion governed by Eq. (38) with timevarying Mach numbers. These results are shown in Fig. 10. The SBRF errors for this test case are 1.4, 2.1, and $2.5 \%$ for lift, moment, and drag, respectively. The accuracy of the SBRF depicted in Fig. 10 indicates that the 200 point OLH resulted in a sufficiently spacefilling data set such that the SBRF can accurately predict at arbitrary inputs. It should be noted that accurate surrogate mapping functions can potentially be generated from much fewer than 200 training cases

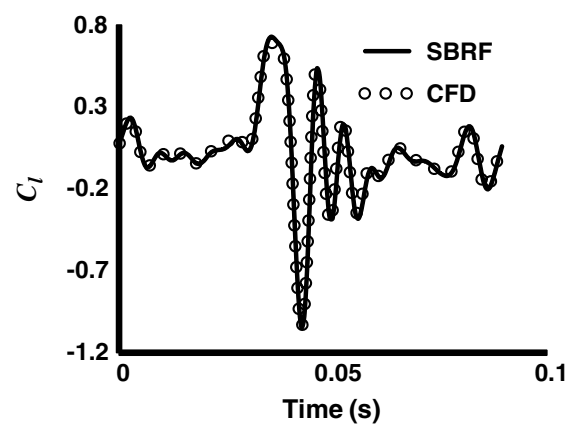

b) Lift coefficient

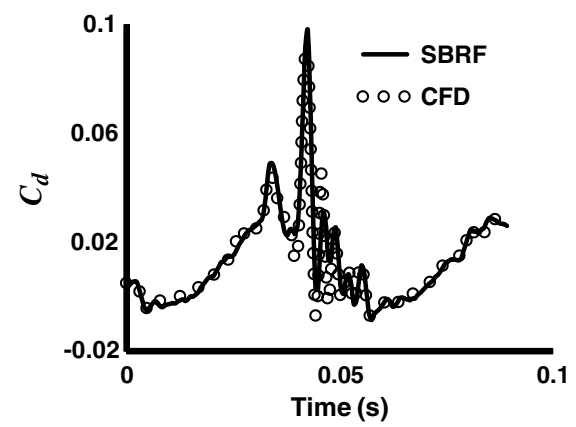

d) Drag coefficient

Fig. 10 Unsteady airloads for multiharmonic pitch input under time-varying Mach number $\left(M_{0}=0.51, \bar{M}=0.22\right.$, and $\left.k_{M}=0.03\right)$. 
by using more "intelligent" sampling methods $[34,42]$ compared to purely space-filling approaches. Such approaches are the subject of ongoing research that is beyond the scope of this paper.

\section{Conclusions}

The reduced-order nonlinear unsteady aerodynamic model described in this paper was shown to effectively mimic CFD predictions of unsteady lift, moment, and drag coefficients for airfoils undergoing simultaneous pitch/plunge oscillations while subjected to fixed and time-varying Mach numbers. The surrogate-based recurrence framework (SBRF) approach to time-domain reducedorder modeling uses kriging surrogates to account for flow nonlinearities, and a recurrence methodology to account for time-history effects associated with unsteadiness. Once constructed from a limited number of full-order CFD analyses, the SBRF predictions require a fraction of a second to compute, while maintaining a level of fidelity corresponding to CFD results that would require several hours to obtain. Therefore, the SBRF approach is appropriate for applications that would require excessive computational resources when using CFD, such as routine coupled trim/aeroelastic response solutions and design optimization. The principal results of this study are as follows:

1) For the pitching airfoil problem considered in this study, an accurate reduced-order unsteady aerodynamic model was generated by the SBRF approach with significantly fewer full-order CFD training cases compared to results reported in the literature for a similar problem in which a proper orthogonal-decomposition-based approach was applied.

2) The effectiveness of the SBRF approach for modeling flow nonlinearities was demonstrated by considering transonic flow conditions. The SBRF accurately modeled the strongly nonlinear effects of moving shocks on the unsteady pitching moments.

3) The SBRF approach accurately modeled unsteady aerodynamic responses associated with time-varying Mach numbers. Therefore, the SBRF is suitable for rotary-wing applications.

\section{Acknowledgments}

This research was sponsored in part by a National Science Foundation (NSF) Alliance for Graduate Education and the Professoriate (AGEP) postdoctoral fellowship for B. Glaz. Partial support from the Center for Rotorcraft Innovation under work breakdown structure 2010-B-1-431-A17, as well as the Vertical Lift Research Center of Excellence (VLRCOE) sponsored by the National Rotorcraft Technology Center (NRTC) with M. Rutkowski as Grant Monitor is also hereby acknowledged.

\section{References}

[1] Leishman, J. G., Principles of Helicopter Aerodynamics, 2nd ed., Cambridge Univ. Press, New York, 2006.

[2] Myrtle, T. F., and Friedmann, P. P., "Application of a New Compressible Time Domain Aerodynamic Model to Vibration Reduction in Helicopters Using an Actively Controlled Flap," Journal of the American Helicopter Society, Vol. 46, No. 1, Jan. 2001, pp. 32-43. doi:10.4050/JAHS.46.32

[3] Jose, A. I., Leishman, J. G., and Baeder, J. D., "Unsteady Aerodynamic Modeling with Time-Varying Free-Stream Mach Numbers," Journal of the American Helicopter Society, Vol. 51, No. 4, Oct. 2006, pp. 299318.

doi:10.4050/JAHS.51.299

[4] Lucia, D. J., Beran, P. S., and Silva, W. A., "Reduced-Order Modeling: New Approaches for Computational Physics," Progress in Aerospace Sciences, Vol. 40, 2004, pp. 51-117. doi:10.1016/j.paerosci.2003.12.001

[5] Hall, K. C., Thomas, J. P., and Dowell, E. H., "Proper Orthogonal Decomposition Technique for Transonic Unsteady Aerodynamic Flows," AIAA Journal, Vol. 38, No. 10, Oct. 2000, pp. 1853-1862. doi: $10.2514 / 2.867$

[6] Thomas, J. P., Dowell, E. H., and Hall, K. C., "Using Automatic Differentiation to Create a Nonlinear Reduced-Order- Model Aerodynamic Solver," AIAA Journal, Vol. 48, No. 1, Jan. 2010, pp. 19-24. doi: $10.2514 / 1.36414$
[7] Lieu, T., and Farhat, C., "Adaptation of Aeroelastic Reduced-Order Models and Application to an F-16 Configuration," AIAA Journal, Vol. 45, No. 6, June 2007, pp. 1244-1257. doi: $10.2514 / 1.24512$

[8] Silva, W., "Identification of Nonlinear Aeroelastic Systems Based on the Volterra Theory: Progress and Opportunities," Nonlinear Dynamics, Vol. 39, 2005, pp. 25-62. doi:10.1007/s11071-005-1907-z

[9] Liu, L., Friedmann, P. P., and Padthe, A. K., "An Approximate Unsteady Aerodynamic Model for Flapped Airfoils Including Improved Drag Predictions," Proceedings of the 34th European Rotorcraft Forum, Liverpool, UK, Sept. 2008.

[10] Trizila, P. C., Kang, C., Visbal, M. R., and Shyy, W., "Unsteady Fluid Physics and Surrogate Modeling of Low Reynolds Number, Flapping Airfoils," 38th Fluid Dynamics Conference and Exhibit, Seattle, WA, AIAA Paper 2008-3821, June 2008.

[11] Trizila, P. C., Kang, C., Visbal, M. R., and Shyy, W., "A Surrogate Model Approach in 2D Versus 3D Flapping Wing Aerodynamic Analysis," 12th AIAA/ISSMO Multidisciplinary Analysis and Optimization Conference, Victoria, British Columbia, AIAA Paper 2008-5914, Sept. 2008.

[12] Suresh, S., Omkar, S. N., Mani, V., and Guru Prakash, T. N., "Lift Coefficient Prediction at High Angle of Attack Using Recurrent Neural Network," Aerospace Science and Technology, Vol. 7, No. 8, 2003, pp. 595-602. doi:10.1016/S1270-9638(03)00053-1

[13] Marques, F. D., and Anderson, J., "Identification and Prediction of Unsteady Transonic Aerodynamic Loads by Multi-Layer Functionals," Journal of Fluids and Structures, Vol. 15, 2001, pp. 83-106. doi:10.1006/jfls.2000.0321

[14] Datta, A., and Chopra, I., "Validation of Structural and Aerodynamic Modeling Using UH-60A Airloads Program Data," Journal of the American Helicopter Society, Vol. 51, No. 1, Jan. 2006, pp. 43-58. doi:10.4050/1.3092877

[15] Datta, A., Nixon, M., and Chopra, I., "Review of Rotor Loads Prediction with the Emergence of Rotorcraft CFD," Journal of the American Helicopter Society, Vol. 52, No. 4, Oct. 2007, pp. 287-317. doi:10.4050/JAHS.52.287

[16] Simpson, T. W., Peplinski, D., Koch, P. N., and Allen, J. K. "Metamodels for Computer-Based Engineering Design: Survey and Recommendations," Engineering with Computers, Vol. 17, 2001, pp. 129-150. doi:10.1007/PL00007198

[17] Queipo, N. V., Haftka, R. T., Shyy, W., Goel, T., Vaidyanathan, R., and Tucker, P. K., "Surrogate-Based Analysis and Optimization," Progress in Aerospace Sciences, Vol. 41, 2005, pp. 1-28. doi:10.1016/j.paerosci.2005.02.001

[18] Forrester, A., Sóbester, A., and Keane, A., Engineering Design via Surrogate Modelling: A Practical Guide, AIAA, Reston, VA, 2008.

[19] Leontaritis, I. J., and Billings, S. A., "Input-Output Parametric Models for Nonlinear Systems," International Journal of Control, Vol. 41, No. 2, 1985, pp. 303-344. doi:10.1080/0020718508961129

[20] Levin, A. U., and Narendra, K. S., "Control of Nonlinear Dynamical Systems Using Neural Networks-Part II: Observability, Identification, and Control," IEEE Transactions on Neural Networks, Vol. 7, No. 1, Jan. 1996, pp. 30-42. doi: $10.1109 / 72.478390$

[21] Xiang, C., "Existence of Global Input-Output Model for Nonlinear Systems," Proceedings of the International Conference on Control and Automation, Budapest, Hungary, June 2005.

[22] Ljung, L., System Identification: Theory for the User, Prentice-Hall, Upper Saddle River, NJ, 1999.

[23] Bisplinghoff, R. L., Ashley, H., and Halfman, R., Aeroelasticity, Addison-Wesley, Reading, MA, 1955.

[24] Mandic, D. P., and Chambers, J. A., Recurrent Neural Networks for Prediction, Wiley, New York, 2001.

[25] Pearson, R. K., "Nonlinear Input/Output Modelling," Journal of Process Control, Vol. 5, No. 4, 1995, pp. 197-211. doi:10.1016/0959-1524(95)00014-H

[26] Simpson, T. W., Lin, D. K. J., , and Chen, W., "Sampling Strategies for Computer Experiments: Design and Analysis," International Journal of Reliability and Applications, Vol. 2, No. 3, 2001, pp. 209-240.

[27] Sacks, J., Welch, W. J., Mitchell, T. J., and Wynn, H. P., "Design and Analysis of Computer Experiments," Statistical Science, Vol. 4, No. 4, 1989, pp. 409-435. doi: $10.1214 / \mathrm{ss} / 1177012413$

[28] Martin, J., and Simpson, T., "Use of Kriging Models to Approximate Deterministic Computer Models," AIAA Journal, Vol. 43, No. 4, 
April 2005, pp. 853-863.

doi: $10.2514 / 1.8650$

[29] Simpson, T. W., Booker, A. J., Ghosh, D., Giunta, A. A., Koch, P. N., and Yang, R. J., "Approximation Methods in Multidisciplinary Analysis and Optimization: A Panel Discussion," Structural and Multidisciplinary Optimization, Vol. 27, No. 5, July 2004, pp. 302-313. doi:10.1007/s00158-004-0389-9

[30] McKay, M. D., Beckman, R. J., and Conover, W. J., "A Comparison of Three Methods for Selecting Values of Input Variables in the Analysis of Output from a Computer Code," Technometrics, Vol. 21, No. 2, May 1979 , pp. $239-245$ doi: $10.2307 / 1268522$

[31] Morris, M. D., and Mitchell, T. J., "Exploratory Designs for Computational Experiments," Journal of Statistical Planning and Inference, Vol. 43, 1995, pp. 381-402.

[32] Jin, R., Chen, W., and Sudjianto, A., "An Efficient Algorithm for Constructing Optimal Design of Computer Experiments," Journal of Statistical Planning and Inference, Vol. 134, No. 1, 2005, pp. 268-287.

[33] Koch, P. N., Evans, J. P., and Powell, D., "Interdigitation for Effective Design Space Exploration Using iSIGHT," Structural and Multidisciplinary Optimization, Vol. 23, No. 2, 2002, pp. 111-126. doi:10.1007/s00158-002-0171-9

[34] Jones, D. R., "A Taxonomy of Global Optimization Methods Based on Response Surfaces," Journal of Global Optimization, Vol. 21, 2001, pp. 345-383. doi:10.1023/A:1012771025575

[35] Lophaven, S. N., Nielsen, H. B., and Søndergaard, J., "DACE-A Matlab Kriging Toolbox, Version 2.0," DTU Informatics, TR IMM-TR2002-12, Lyngby, Denmark, 2002.
[36] Peroomian, O., Chakravarthy, S., Palaniswamy, S., and Goldberg, U., "Convergence Acceleration for Unified-Grid Formulation Using Preconditioned Implicit Relaxation," AIAA Paper 98-0116, Reno, NV, Jan. 1998.

[37] Peroomian, O., Chakravarthy, S., and Goldberg, U., "A 'GridTransparent' Methodology for CFD,” AIAA Paper 97-0724, Reno, NV, Jan. 1997.

[38] Chakravarthy, S. R., and Osher, S., "New Class of High Accuracy TVD Schemes for Hyperbolic Conservation Laws," 23rd AIAA Aerospace Sciences Meeting and Exhibit, Reno, NV, AIAA Paper 85-0363, Jan. 1985.

[39] Attar, P. J., Dowell, E. H., White, J. R., and Thomas, J. P., "Reduced Order Nonlinear System Identification Methodology," AIAA Journal, Vol. 44, No. 8, Aug. 2006, pp. 1895-1904. doi:10.2514/1.16221

[40] Rogers, K. L., "Airplane Math Modeling Methods for Actively Control Design," AGARD CP-228, Neuilly-sur-Seine, France, Aug. 1977.

[41] Rodden, W. P., and Albano, E., "A Doublet-Lattice Method for Calculating Lift Distributions on Oscillating Surfaces in Subsonic Flows," AIAA Journal, Vol. 7, 1969, pp. 279-285. doi: $10.2514 / 3.5086$

[42] Sasena, M. J., Papalambros, P., and Goovaerts, P., "Exploration of Metamodeling Sampling Criteria for Constrained Global Optimization," Engineering optimization, Vol. 34, 2002, pp. 263-278. doi:10.1080/03052150211751
K. Willcox Associate Editor 